\title{
PROLACTINOMAS E GRAVIDEZ
}

\author{
MANUELA CARVALHEIRO, ISABEL FAGULHA, MARGARIDA MOREIRA, \\ ELIZABETE GERALDES, FRANCISCO CARRILHO, MARGARIDA BASTOS, ANA FAGULHA \\ ISABEL PAIVA, ALMEIDA RUAS \\ Serviço de Endocrinologia. Serviço de Obstetrícia. Hospitais da Universidade de Coimbra. Coimbra.
}

\begin{abstract}
RESUMO
Os autores, após uma breve introdução teórica sobre a repercussão dos prolactinomas no eixo hipotálamo - hipófise - gónadas, nomeadamente no que respeita à esterilidade, falam da sua experiência no tratamento destas situaçð̃es com a bromocriptina. São analizadas 10 gravidezes em 8 doentes, descrevendo-se o protocolo e terapêuticas utilizadas durante a gestação. A terapêutica com a bromocriptina foi mantida em 6 doentes e suspensa em 4 após a fecundação, tendo ambos os grupos resultados idênticos e satisfatórios, pelo que se conclui ser desnecessária a atitude cautelosa da manutenção da bromocriptina durante a gravidez. É descrita e discutida uma situação de cura após a gravidez. Por último reafirma-se a posição sempre defendida da manutenção da terapêutica médica com bromocriptina, até à cura dos prolactinomas, mesmo nas mulheres que não desejam engravidar.
\end{abstract}

\section{SUMMARY}

\section{Prolactinomas in the course of pregnancy}

Twenty women with prolactinomas received bromocriptine treatment which resulted in 10 term pregnancies. Bromocriptine was continued in 6 patients and stopped in the last 4 , as soon as pregnancy was established. The pregnancies in both series, were clinically uneventful. No case of congenital malformations or abnormalities in the babies was reported. Thus, the pregnancy does not worsen the condition and some cases of benefit are described ( 1 in our serie). After pregnancy and lactation bromocriptine treatment must be continued in hyperprolactinaemic patients till resolution.

\section{INTRODUÇ̃̃o}

As situações de hiperprolactinémias, pela sua repercussão a nível do eixo hipotálamo-hipófise-gónadas, constituem uma das causas conhecidas de esterilidade conjugal. Nas mulheres, para além de ciclos anovulatórios existem muitas vezes galactorreia e irregularidades menstruais, que podem ir até à amenorreia.1-5 No homem, as manifestações clínicas habituais são de impotência sexual, esterilidade com oligo ou azoospermia e galactorreia. 5

Cerca de metade destas situações devem-se à existência de tumores produtores de prolactina - prolactinomas que na maioria das vezes são microadenomas (diâmetro $<10 \mathrm{~mm}$ ) só visíveis à tomografia axial computorizada craneoencefálica. Há no entanto prolactinomas de maiores dimensões (diâmetro $>10 \mathrm{~mm}$ ), macroprolactinomas, alguns dos quais com expansão supra e/ou infrasselar que se acompanham de lesão do quiasma com alteração dos campos visuais, e quadros de hipertensão intracraneana.1, 2, 5, 6

O conhecimento da acção da dopamina a nível do controlo de produção de PRL (controlo inibidor) 5, 7, 11 e a descoberta de fármacos dopaminérgicos potentes, como a bromocriptina, vieram permitir a resolução médica destas situaçőes, embora a terapêutica, tenha de se prolongar durante anos, no que respeita à possível cura das lesões.8, 10, 11, 14
Outra abordagem terapêutica é a microcirurgia transfenoidal, método de primeira linha em macroadenomas com repercussão neurológica e compromisso visual.1, 12 No caso dos microadenomas, e nos macro não expansivos, em mulheres que desejam procriar, a terapêutica médica é preferencial, por permitir a resolução segura da esterilidade.1, 12, 25

A gravidez não é no entanto desprovida de riscos já que é em si mesmo uma situação geradora de hiperplasia hipofisária. 1, 12, 15-18 Os quadros de expansão tumoral, inicialmente temidos, têm sido felizmente raros, e hoje considera-se que a gravidez e a lactação não acarretam obrigatóriamente um agravamento, estando até já descritos casos de melhoria, e até cura no pós-parto.1, 12, 16-18

E no entanto um procedimento obrigatório, a pesquisa periódica de sinais e sintomas de expansão selar durante a gestação. Caso tal expansão se verifique, a reintrodução da bromocriptina é muitas vezes terapêutica suficiente.1, 12, 20 Em situações mais graves de hipertensão intra-craniana, ou alteração súbita à campimetria, a microcirurgia transfenoidal de urgência está indicada.1, 12

Temos tido desde há muito, um interesse especial por estas situações, encontrando-se em tratamento neste momento 25 doentes com prolactinoma, 5 do sexo masculino e 20 do sexo feminino. O número de gravidezes ocorridas neste grupo foi de 10 em 8 doentes. 


\section{DOENTES E MÉTODOS}

Estudámos e seguimos, 10 gestações em 8 mulheres de raça branca, com idade compreendida entre os 25 e os 31 anos. Todas apresentavam galactorreia e 7 estavam amenorreicas (Quadro 1). Uma das doentes, tinha ciclos menstruais regulares. Os ciclos eram anovulatórios, sendo a infertilidade uma queixa comum.

No que respeita a paridade, 4 (casos 1 a 4) eram nuliparas tendo as restantes (casos 5 a 8) várias gestações anteriores (Quadro 1, coluna Gestação/Paridade).

A duração das queixas variava de 2 a 11 anos.

Os estudos radiológicos efectuados, mostraram em 2 casos, alterações à radiografia simples do crânio, com imagens de duplo soalho, e em dois casos, alargamento da sela turca com destruição do soalho. Não nos foi possível obter o R.X. do crânio de 2 doentes (Casos 6 e 8) que nos foram enviadas já grávidas por outras Instituições de Saúde. As TAC craneoencefálicas, realizadas na DIATON em Coimbra, mostraram microadenomas, que em 3 doentes se acompanhavam de sela turca vazia. Só num caso havia alteração à campimetria com aperto dos sectores temporais.

Os doseamentos hormonais plasmáticos foram feitos no Laboratório de Hormonologia da Faculdade de Medicina de Coimbra por método radioimunológico. Os valores da PRL, variavam antes do início da terapêutica entre 51 a $246 \mathrm{ng} / \mathrm{ml}$, valor médio $\pm 145 \mathrm{ng} / \mathrm{ml}$.

O eixo hipotálamo-hipófise-gónadas foi estudado (prova do LH-RH; $\mathrm{E}_{2}$ e progesterona), sendo de salientar um acentuado hipoestrogenismo nas mulheres amenorreicas. As restantes linhas hipofisárias não mostraram alterações.

Nenhuma das doentes tomava ou tinha tomado fármacos capazes de induzir hiperprolactinémias.
A terapêutica médica com bromocriptina foi instituída em doses variáveis e progressivas $(2,5$ a $12,5 \mathrm{mg} / \mathrm{dia}$, dose média $\pm 10 \mathrm{mg} /$ dia), sempre com monitorização da PRL. A dose, era variável e ajustada caso a caso, de acordo com os níveis hormonais plasmáticos tendo sempre em conta a sua normalização.

A regularização dos ciclos menstruais ocorreu em média 3 meses, após o início da bromocriptina, e a gravidez entre 2 a 19 meses (tempo médio \pm 7 meses). Duas doentes tiveram uma segunda gravidez, sob terapêutica (casos 3 e 6) que decorreu após um intervalo de tempo respectivamente de 44 e 17 meses. Os ciclos menstruais foram nesse intervalo de tempo ovulatórios, mas as doentes utilizaram métodos anticoncepcionais que suspenderam ao desejarem nova gestação.

As primeiras 6 doentes (casos 1 a 6 ) tratadas entre 1981/1983, mantiveram a terapêutica com bromocriptina durante a gravidez, em doses variáveis de 2,5 a $15 \mathrm{mg} /$ dia, ajustadas caso a caso (dose média $10 \mathrm{mg} / \mathrm{dia}$ ). Os bons resultados obtidos, os avanços da literatura e a experiência de outros centros, levou-nos a mudar de atitude, o que adiante discutiremos, e de 1983 a 1985, nos restantes 4 casos (7 a 10) a terapêutica médica foi suspensa após fecundação.

Em ambos os grupos a monitorização da PRL, foi feita mensalmente. As doentes do último grupo (s/bromocriptina) amamentaram, tendo-se doseado a PRL periodicamente, antes e após as mamadas. Durante a gravidez as doentes fizeram campimetrias mensais no Serviço de Oftalmologia dos H.U.C.

A observação obstétrica, foi idêntica à das gravidezes normais, e decorreu em simultâneo com a observação endocrinológica.

$\mathrm{Na}$ altura do parto, foram feitas colheitas para doseamento de PRL na mãe e no sangue do cordão. No pós-parto as doentes foram reavaliadas sob o ponto de vista bioquimico, oftalmológico e radiológico.

QUADRO 1 Dados clínicos, radiológicos e valores de PRL em 10 casos de prolactinomas

\begin{tabular}{|c|c|c|c|c|c|c|c|c|c|c|}
\hline Casos & Idade & Menarca & $\begin{array}{l}\text { Gestação } \\
\text { paridade }\end{array}$ & Sintomas & $\begin{array}{l}\text { Duração dos } \\
\text { sintomas } \\
\text { em anos }\end{array}$ & TAC & $\begin{array}{c}\text { Tumor } \\
\text { diagnosticado } \\
\text { por Rx S.T }\end{array}$ & $\begin{array}{l}\text { PRL } \\
\text { Basal } \\
\mathrm{ng} / \mathrm{ml}\end{array}$ & Campimetria & $\begin{array}{l}\text { Fundos } \\
\text { oculares }\end{array}$ \\
\hline 1 & 26 & 11 & 0 & $\begin{array}{l}\text { Amenorreia } \\
\text { Secundária } \\
\text { + Galactorreia }\end{array}$ & 3 & Microadenoma & $\begin{array}{l}\text { Duplo } \\
\text { Soalho } \\
\text { Fundo }\end{array}$ & 275 & N & $\mathbf{N}$ \\
\hline 2 & 27 & 11 & 0 & $\begin{array}{l}\text { Amenorreia Sec } \\
\text { + Galactorreia } \\
\text { + Pert. visuais }\end{array}$ & 4 & $\begin{array}{l}\text { Sela turca } \\
\text { vazia } \\
\text { Microadenoma }\end{array}$ & $\begin{array}{c}\text { Sela turca } \\
\text { aumentada c/ } \\
\text { destruição do } \\
\text { soalho }\end{array}$ & 82 & $\begin{array}{l}\text { Aperto discreto } \\
\text { nos sectores } \\
\text { temporais }\end{array}$ & $\mathbf{N}$ \\
\hline 3 & 25 & 13 & 0 & $\begin{array}{l}\text { Amenorreia Sec } \\
\text { + Galactorreia }\end{array}$ & 6 & Microadenoma & $N$ & 250 & $\mathrm{~N}$ & $N$ \\
\hline 4 & 31 & 14 & 0 & $\begin{array}{l}\text { Amenorreia Sec } \\
\text { + Galactorreia }\end{array}$ & 7 & $\begin{array}{l}\text { Microadenoma } \\
\text { Sela turca vazia }\end{array}$ & $\begin{array}{c}\text { Sela turca } \\
\text { aumentada c/ } \\
\text { destrução soalho }\end{array}$ & 62 & N & $\mathrm{N}$ \\
\hline 5 & 32 & 12 & $3 \mathrm{G} / 2 \mathrm{P}$ & $\begin{array}{l}\text { Amenorreia Sec } \\
\text { + Galactorreia }\end{array}$ & 7 & $\begin{array}{l}\text { Aracnoidocelo } \\
\text { Sela turca vazia } \\
\text { supeita de } \\
\text { microadenoma }\end{array}$ & $N$ & 51 & $\mathbf{N}$ & $\mathbf{N}$ \\
\hline 6 & 25 & 14 & $1 \mathrm{G} / 0 \mathrm{P}$ & Galactorreia & 5 & Microadenoma & - & 76 & $\mathbf{N}$ & $\mathrm{N}$ \\
\hline 7 & 27 & 12 & $2 \mathrm{G} / 1 \mathrm{P}$ & $\begin{array}{c}\text { Amenorreia Sec } \\
\text { + Galactorreia }\end{array}$ & 6 & Microadenoma & Duplo soalho & 108 & $\mathrm{~N}$ & $\mathrm{~N}$ \\
\hline 8 & 31 & 14 & $2 \mathrm{G} / 1 \mathrm{P}$ & $\begin{array}{l}\text { Amenorreia Sec } \\
+ \text { Galactorreia }\end{array}$ & 8 & Microadenoma & - & 52 & $\mathbf{N}$ & $\mathrm{N}$ \\
\hline 9 & \multicolumn{10}{|c|}{ 2. ${ }^{a}$ gravidez do caso $n .^{\circ} 3$} \\
\hline 10 & \multicolumn{10}{|c|}{ 2..$^{a}$ gravidez do caso n. 6} \\
\hline
\end{tabular}




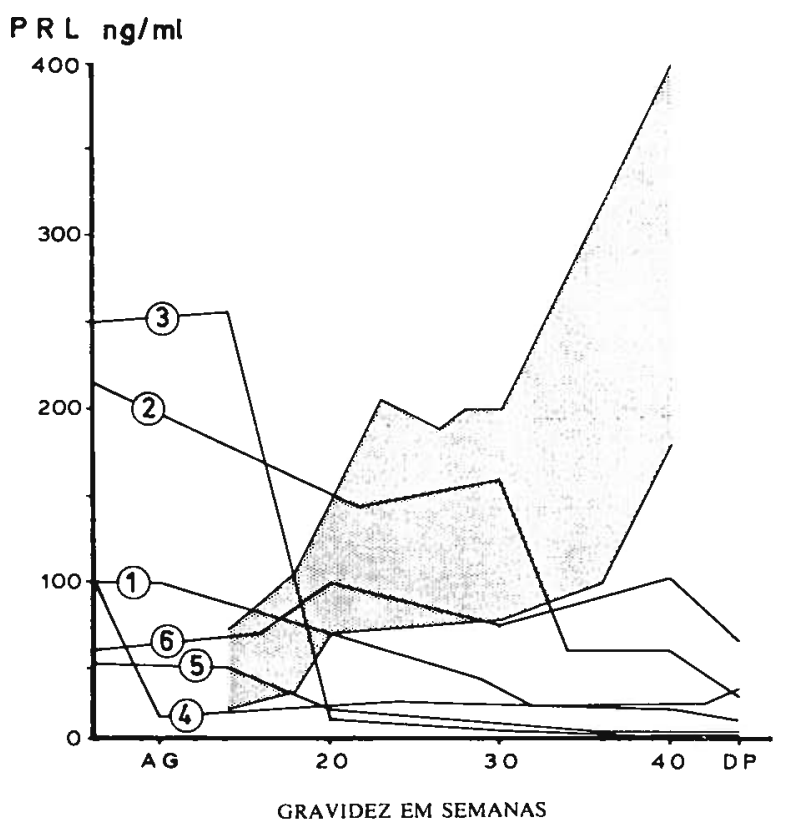

Figura 1: Valor médio dos PRL nas diferentes semanas gestacionais nas doentes que mantiveram a bromocriptina durante a gravidez (casos 1 a 6 ).

\section{RESULTADOS}

Os níveis de PRL durante a gravidez, e tendo em conta as respectivas idades gestacionais, mantiveram-se baixos no grupo a tomar bromocriptina, e dentro dos valores normais no grupo sem bromocriptina (Fig. 1 e 2).

Não se verificou em qualquer dos grupos, com e sem bromocriptina, agravamento da lesão hipofisária, confirmada no pós-parto, por campimetria e exames radiográficos (R.X. do crânio e em algumas doentes TAC craneoencefálica. O caso 4, em que havia erosão do soalho está em franca reconstrução óssea. Dois casos de microadenoma (l e 6) mostram agora à TAC, uma hipófise normal (Quadro 2).

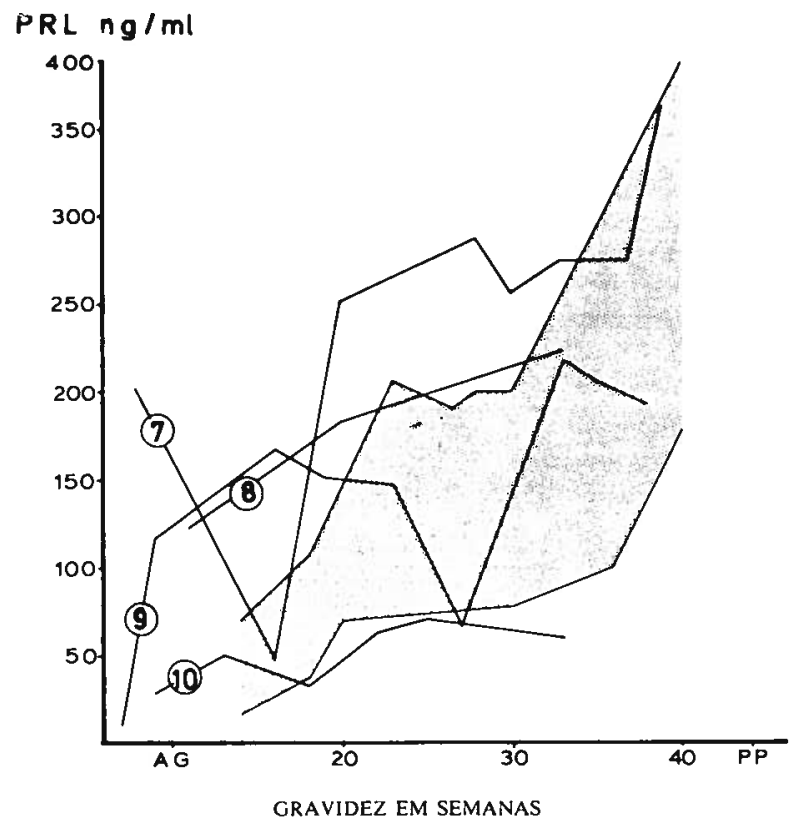

Figura 2: Valor médio de PRL, nas diferentes semanas de gravidez, nas doentes que suspenderam a bromocriptina durante a gravidez (casos 7 a 10).

A terapêutica médica manteve-se contínua no $10^{\circ}$ grupo, embora com ajustamento da dose no pós-parto e sempre com monitorização da PRL.

Nas doentes do segundo grupo (sem bromocriptina), a reintrodução do medicamento foi feita após a lactação, face aos valores elevados de PRL que apresentavam 119 a $417 \mathrm{ng} / \mathrm{ml}$ - média $295 \mathrm{ng} / \mathrm{ml}$. Em nenhum caso houve menstruação espontânea.

Nas doentes que não mostraram desejo de nova gravidez foi feita anticoncepção com DIU, e num caso estro-progestativo de baixa dosagem.

Uma das doentes (caso 6) tratada com bromocriptina durante a gravidez, suspendeu a terapêutica por intolerância,

\section{QUADRO 2 Evolução no pós-parto}

\begin{tabular}{|c|c|c|c|c|c|c|c|}
\hline Casos & Amamentaçãoo & $\begin{array}{c}\text { P'KL, Nlaullada } \\
\mathrm{ng} / \mathrm{mI}\end{array}$ & Bromocriptina & Anticoncepção & $\mathrm{TAC} / \mathrm{Rx}$ & Campimetria & Observaçðes \\
\hline 1 & $\mathbf{N}$ & - & Imediata & DIU & Normal & $\mathbf{N}$ & \\
\hline 2 & $\mathrm{~N}$ & - & Imediata & DIU & $\begin{array}{l}\text { Sela turca } \\
\text { vazia } \mathrm{c} / \mathrm{microa}- \\
\text { denoma }\end{array}$ & $\mathbf{N}$ & \\
\hline 3 & $\mathrm{~N}$ & - & Imediata & DIU & - & $\mathbf{N}$ & $\begin{array}{l}\text { 2. }{ }^{\text {a }} \text { gravidez } \\
\text { em } 1984(9)\end{array}$ \\
\hline 4 & $\mathrm{~N}$ & - & Imediata & DIU & $\begin{array}{l}\text { Reconstruçăo } \\
\text { óssea da sela } \\
\text { turca (Rx) }\end{array}$ & & \\
\hline 5 & $\mathrm{~N}$ & - & Imediata & DIU & - & $\mathrm{N}$ & \\
\hline 6 & $\mathrm{~N}$ & - & Suspendeu & & Normal & & $\begin{array}{l}\text { 2." gravidez } \\
\text { 1984/1985 } \\
\text { (em curso) }\end{array}$ \\
\hline 7 & $4 \mathrm{M}$ & $\begin{array}{c}\text { Antes - } 245 \\
\text { Após - } 471\end{array}$ & Iniciou após $9 \mathrm{M}$ & DIU & Microadenoma & $\mathbf{N}$ & \\
\hline 8 & $8 \mathrm{M}$ & $\begin{array}{l}\text { Antes - } 119 \\
\text { Após - } 128\end{array}$ & Iniciou após $8 \mathrm{M}$ & $\begin{array}{l}\text { Estroprogestativo } \\
\text { mini doseados }\end{array}$ & & $\mathbf{N}$ & \\
\hline 9 & $1 \mathrm{M}$ & $\begin{array}{l}\text { Antes - } 118 \\
\text { Após - } 125\end{array}$ & Iniciou após $1 \mathrm{M}$ & & & & \\
\hline
\end{tabular}


passado 1 ano. Os valores da PRL, mantiveram-se normais, (valor médio $\pm 20 \mathrm{ng} / \mathrm{ml}$ ), ciclos menstruais regulares e ovulatórios e sem galactorreia. A TAC da sela turca de controlo, realizada neste periodo mostrou uma hipófise normal (havia microadenoma; Quadros 1 e 2). A doente voltou a engravidar passados 3 meses da suspensão de bromocriptina (caso 10) e, durante a 2. ${ }^{2}$ gestação, agora no seu terminus, os valores de PRL têm-se mantido sempre dentro da normalidade. Esta doente, pode ser considerada um caso de cura após a gravidez, atendendo a que foi na sequência desta que se verificou a normalização clínica, bioquímica e radiológica.

No que respeita à evolução da gravidez sob o ponto de vista obstétrico, as $\mathbf{1 0}$ gestações decorreram sem problemas (uma está ainda em curso).

Os partos foram eutócicos e de termo.

Os recém-nascidos pesavam entre 2,950 e $3,850 \mathrm{Kg}$. Nenhuma situação de morbilidade peri ou neo-natal foi detectada, nomeadamente SDR (síndrome de deficiência respiratória) mesmo nos casos em que a terapêutica com a bromocriptina foi mantida durante a gestação. Estas crianças, com idades compreendidas entre 3 meses e 5 anos, têm sido sempre vigiadas por pediatras, não havendo a assinalar quaisquer alterações no desenvolvimento estato-ponderal e/ou psico-motor.

Foi feito aleitamento artificial nos filhos das mulheres tratadas com bromocriptina durante a gravidez.

$O$ outro grupo (sem bromocriptina) amamentou normalmente por períodos variáveis de 1 a 8 meses. As doentes deixaram de amamentar, por insuficiência do leite materno face às necessidades nutricionais dos recém-nascidos. Os doseamentos de PRL durante a lactação mostraram variabilidade (Quadro 2) mantendo-se dentro dos parâmetros aceitáveis para a situação.

\section{DISCUSSÃO}

A utilização da bromocriptina como indutor da ovulação, no caso dos prolactinomas ou outras situações de hiperprolactinémia, está hoje universalmente aceite como método terapêutico eficaz $(80$ a $100 \%)$ e seguro. ${ }^{1-3,9-12}$

Nas nossas 8 doentes, a eficácia foi de $100 \%$, sendo de salientar o curto intervalo de tempo que esteve entre o início do tratamento, a regularização dos ciclos menstruais $( \pm 3$ meses) e a fecundação ( 2 a 19 meses).

Embora se saiba que o fármaco atravessa a barreira placentar não são conhecidos, até ao momento, efeitos teratogénicos.1, 10, 12, 14 Nos filhos das mulheres que tomaram bromocriptina durante a gravidez, têm no entanto sido descritos casos de SDR (síndrome de deficiência respiratória) e baixo peso ao nascer.13, 24 Alguns autores têm defendido que tais situações mórbidas serão consequência de possíveis efeitos nocivos, de hipoprolactinémia materna e fetal, induzida farmacológicamente. $13,21,24 \mathrm{Na}$ nossa série, as mulheres tratadas com bromocriptina durante a gestação (casos $1 \mathrm{e}$ 6), apresentaram valores de PRL francamente baixos à altura do parto (Fig. 2), mas todos os recém-nascidos eram normais, pelo que pensamos que outros factores terão que estar necessáriamente implicados.

Os estudos efectuados durante a gravidez e a lactação no que respeita à possivel expansão tumoral, e a experiência de vários centros sobre o assunto, vieram mostrar ser desnecessária a atitude cautelosa da manutenção da bromocriptina durante a gravidez, com obrigatoriedade de aleitamento artificial.1, 12, 15-18, 24 Nós próprios abandonámos essa atitu- de, sem que, daí resultasse qualquer deterioração no estado das doentes.

Por outro lado, fala-se hoje de um provável efeito benéfico de gravidez nos prolactinomas e cada vez se descrevem mais curas após a gestação.1, 12, 15, 24 A explicação fisiopatológica desta evolução ainda não está completamente esclarecida, mas sugerem-se alterações de tipo isquémico inerentes às variações do volume hipofisário próprias da gravidez, que conduziriam a enfarte ou apoplexia do adenoma. As alterações da dinâmica hormonal durante a gestação, nomeadamente de relação estrogéneo/PRL, e a queda brusca dos níveis hormonais após o parto, podem de alguma maneira intervir na evolução favorável de alguns prolactinomas. 12, 24 $\mathrm{Na}$ nossa série já referimos um caso de cura após a gravidez, (caso 6) que desenvolvemos detalhadamente ao analisar os resultados. Este caso reveste-se de um interesse particular, por a doente já na fase de cura ter engravidado de novo, mantendo sempre valores de PRL normais de acordo com a idade gestacional (caso 10; Quadro 2).

$\mathrm{Na}$ maioria das vezes, no entanto, as situações de hiperprolactinémia com prolactinoma, mantêm-se após a gravidez, o que levanta o problema ainda hoje controverso da manutenção de terapêutica médica com bromocriptina, mesmo nas mulheres que não desejam nova gestação. 1, 12, 22 Embora o que se conhece da história natural dos prolactinomas, até porque maioritariamente microadenomas não nos leve a recear evoluções expansivas, o desarranjo hormonal mantem-se e como tal deve ser tratado e curado se possível. $1,12,14$

A bromocriptina administrada de forma contínua consegue para além da normalização funcional, reduzir em mais de $50 \%$ o tamanho do tumor, mesmo nos macroadenomas, com remineralização da sela turca em caso de destruição óssea (caso 4; Quadro 2). A inócuidade do fármaco permite a manutenção da terapêutica ao longo dos anos (2 a 7 anos) conduzindo em grande número de casos à cura do adenoma, por um mecanismo que se pensa ser de tipo necrótico por acção directa da bromocritpina.1, 12, 14, 23 a nível tumoral.

Outra alternativa terapêutica, é a microcirurgia transfenoidal, que em relação à bromocriptina tem a vantagem de uma maior percentagem de curas (50 a $60 \%$ ) em menor espaço de tempo.1, 2, 12, 25 Mas a cirurgia não é isenta de riscos 1, 2, 25 e a nossa pouca experiência neste campo, leva-nos a ser cautelosos na mudança de uma atitude terapêutica, (a terapêutica médica com a bromocriptina) que até ao presente tem trazido resultados francamente positivos.

\section{BIBLIOGRAFIA}

1. BERGHT: Prolactinomas and Pregnancy - The Proceedings of a Special Symposium held at the XI th World Congress ou Fertility and Sterility, Dublin, June 1983. MTP Press Limited.

2. REICHLIN SEYMOUR, M. D.; P. H. D.: The Prolactinoma Problem. N. Engl. J. Med., 1979; 300: 313-315.

3. MOORE, D. M.; BUCKINGHAM, M. S.; EINGH, M. M.; MILLIGAN, M. P.; ELSTEIN M.: Serum prolactin in female infertility. Lancet $1978 ; 2: 1243-1245$.

4. KREDENTSER, J. V.; HOSKINS, C. F.; SCOTT, J. 2.: Hyperprolactinemia: a significant factor in female infertility. Am. J. Obste. Gynecol., 1981; 139: 264-267.

5. WILLIAMS: Textbook cf. Endocrionology. Philadelfia: W B SAUNDESS 1981 
6. HEMMINGHY, H. S.; KALKHOLFF, M.; DANIELS, K.; WILLNANDES, D. L.; GRAPEN, A. L.; HANGTTEN, J. P.: Computed tomographic study of hormone-secreting microadenomas.

7. FRANTZ, ANDREW 6. Prolactin: N. Engl J. Med., 1981; 298: 201-207.

8. BRESSIAN, D.; BRANDI, A. N.; MARTRES, M. P.: et al. Dopaminergic receptors in human prolactin secreting adenomas: a quantitative study. $J$. Clin Endocrinol., 1980; 51 : 1037-1043 .

9. STRUCH, G.; BRICAIREH: Dynamique des modifications hormonales induite par la bromocriptine au cours des hiperprolactinemis: La Nouvelle Presse Medical, 1977; 6: 175-179.

10. VANCE, M. L.; EVANS, W. S.; THORNER, M. O.: Bromocriptine. Ann Intern. Med., 1984; 100: 78-91.

11. LINQUETTE, M.; FOSSATI, P.: Hyperprolactinismes et Anti-Prolactinismes. Sem. Hop. Paris, 1977; 24: 1431-1438.

12. MARK, E.; MOLITCH, M. D.: Pregnancy and the hyperprolactinemic Woman.: N. Engl. J. Med., 1985; 312: 1364-1369.

13. HANTH, J.; RECHARD, PARKER, MAC DONALD PAUL, POSTER JOHN, JOHNTEN JOHN: A role of-fetal prolactin in lung maturation. Obst. and Gynecology, 1978; 51: 81-88.

14. JOHNSTEN, D. G.; HALL, K.; KENDALL - TAYLOR, P.; PATRICK, D.; WATSEN, M.; LOOK, D. B.: Effect of dopamine agonist Withdrawal after long - term therapy in prolactinomas: studies with high-definition computed tomography: Lancet 1984; 2 : 187-92.

15. RJOSKHK, FAHLBUSH, R.; AND VON WERDER, K.: Influence of pregnancies on prolactinomas. Acta Endocrinol., (Copenh) 1982; 100: 332-346.

16. MAGYAR, D. M.; MARSHALL, J. R.: Pituitary tumors and pregnancy: Am. J. Obstet, Gynecol., 1978; 132: 739-51.

17. GENZELL, C.; WANG, C. F.: Outcome of pregnancy in Women with pituitary adenoma.: Fertil steril, 1979; 31: 363-72.

18. ZARATE ARTUR, CANETES \& ELIAS, ALGER MUCC, FASSBACH GERARDE: The effect of Pregnancy and lactation on pituitary prolactin secreting tumours. Acta Endocrinológica, 1979; 92: 407-411.
19. THORNER, M. O.; EDWARDS, C. R.; WARTH, M.; CHARLES, DACIE, J. E.; MOULT, P. J.; MEES, L. H.; JENE, A. E.; BESSER, G. M.: Pregnancy in patients presenting with hiperprolactinaemia. British medical journal, 1979; 2: $771-774$.

20. VAN, MOEN, E.; VAN DER VIGVEN, J. C. M.; GUERRETSEN, G.; HEKSTER, M. E. M.; WATTENDORFF, M. A.: Rapid regression of a suprasselar extending prolactinoma after bromocriptine treatment during pregnancy. Fertil Steril, 1981; 36: 173-177.

21. JIMENER SCHULTZ; JAHNSTEN: Fetal lung maturation. III Amniotic fluid phosphatidic acid phosphohydrolase (PA Pase) and its relation to the lecithin/sphingomyelin ratio. Obstetric-Gyne., 1976; 46: 580-590.

22. MARCH, C. M.; KLETZKY, O. A.; DAVAJAN, V.; TEAL, J.; WEISS, M.; APUZZO, M. L. J.; MANS, R. P.; MISHEL, D. R.: Longitudinal evolution of patients with untreated prolactin-secreting pituitary adenoma. Am. J. Obstet. Gynecol., 1981; 139: 835-844.

23. SOBRINHO, L. G.; NUNER, M. C.; CALHAR - JERGE, C.; MAURICO, J. C.; SANTS, M. A.: Effect of treatment with bromocriptine on the size and activity of prolactin producing pituitary tumours. Acta Edocrinol., (Copenh.) 1981; 96: 24-29.

24. NYBOR ANDERSON; STARNG, Y.; TABOR, ANNE, KALUND, H.; YENSEN, AND WESTERGAARD, J. C.: The possible prognostic value of serum prolactin increment during pregnancy in hyperprolactineamic patients. Acta Endocrinologica, (Copenh.) 1983; 102: 1-5.

25. ZERVES, N. T.: Surgical results for pituitary adenomas: results of an international survey In: Secretory tumors of the pituitary gland. New York: Maven Press., 1984: 377-81.

Pedido de separatas: Manuela Carvalheiro Serviço de Endocrinologia Hospitais da Universidade de Coimbra 3000 Coimbra. Portugal. 